\title{
Characterization of a novel gene related to antibiotic susceptibility in Pseudomonas aeruginosa
}

\begin{abstract}
Lixin Shen ${ }^{1}$, Yanling $\mathrm{Ma}^{1}$ and Haihua Liang ${ }^{1,2}$
Pseudomonas aeruginosa is a human pathogen with increased intrinsic resistance to a large number of antibiotics used in clinical therapy. Therefore, understanding the mechanisms of resistance and developing therapy alternatives for $P$. aeruginosa are of profound importance. Previous work from our laboratory demonstrated that several mutants have isolated with altered expression of the phzA1B1C1D1E1F1G1 (phzA1) operon in the presence of sub-inhibitory concentrations (SICs) of tetracycline (TET). The present study investigates the roles of the PA0011 gene in mediating phzA1 expression at SIC of TET. The PAO011 gene encodes $2-\mathrm{OH}$-lauroytransferase by controlling the synthesis of the cell envelope and the outer membrane. We found that the PA0011 mutant strain was susceptible to several different antibiotics and environmental stresses. Complementation in the PA0011 mutant restored these phenotypes to wild-type levels. In addition, expression of the PAO011 gene, as monitored through a luciferase reporter, is increased at SICs of antibiotics. Indeed, the expression of the PAOO11 gene increased about threefold in $p q s R$ and $p q s H$ mutants compared with the wild-type PAO1. However, the PA0011 gene negatively regulates the quorum sensing (QS) system. Taken together, these data suggest that PA0011 is involved in susceptibility to antimicrobial agents in $P$. aeruginosa, and that its susceptibility effect maybe partly dependent on increased QS expression.
\end{abstract}

The Journal of Antibiotics (2012) 65, 59-65; doi:10.1038/ja.2011.111; published online 7 December 2011

Keywords: PA0011; P. aeruginosa; quorum sensing system; SICs; tetracycline

\section{INTRODUCTION}

Pseudomonas aeruginosa is naturally more resistant to antibacterial agents than many other Gram-negative species of bacteria, including Escherichia coli. ${ }^{1}$ The growing problem of antibiotic resistance among bacterial pathogens and the escalating difficulty in finding new antibiotics are driving the search for a new approach to antibacterial chemotherapy. ${ }^{2}$ There are many resistance mechanisms to antibiotics that exist in nature and in $P$. aeruginosa. A number of non-essential bacterial proteins may potentially contribute to intrinsic resistance. The susceptibilities of bacteria to antibiotics depends on many factors, including the structure and the composition of the cell envelope, the presence of inactivating enzymes and the availability of efflux pumps. $^{3-5}$ All these mechanisms are potentiated by the relative impermeability of the outer membrane to both hydrophilic and hydrophobic drugs. ${ }^{6}$ This outer membrane property in P. aeruginosa is mostly dependent on the structural heterogeneity of porin OprF, as only a small proportion of this protein forms through the lipid bilayer. $^{7}$ The smaller antibiotics, such as erythromycin and novobiocin, cannot efficiently cross the cell envelope via the porin uptake pathway. ${ }^{1}$ Lipopolysaccharides (LPS) are the major components on the outer surface of the outer membranes of Gram-negative bacteria, including $P$. aeruginosa and E. coli, and form a barrier around the cell. ${ }^{8,9}$ Some low-MW compounds can penetrate the outer membrane via porins, but larger, potentially harmful agents, including many antibiotics, are excluded. ${ }^{10}$ As with antimicrobials, extensive use has led to increasing quinolone resistance among bacterial pathogens. Resistance to fluoroquinolones by $P$. aeruginosa is often mediated by mutations in target enzymes or upregulation in efflux pump expression, coupled with intrinsically low outer membrane permeability. ${ }^{11,12}$

Quorum sensing (QS) is a global regulatory system controlling population behavior, and has a fundamental role in bacterial pathogenicity. ${ }^{13}$ There are three intertwined QS systems in P. aeruginosa: the acyl-homoserine lactone (HSL) mediated las and $r h l$ systems, and the 2-alkyl-4(1 H)-quinolone (AHQ) signal-based system. ${ }^{14-17}$ The las and $r h l$ systems consist of the transcriptional activators LasR and RhlR, and the signal synthases LasI and RhlI, respectively. The signals in the las and rhl systems are $\mathrm{N}$-(3-oxododecanoyl)-HSL (3oxo- $\mathrm{C}_{12}$-HSL) and N-butyryl-HSL $\left(\mathrm{C}_{4}\right.$-HSL), respectively. ${ }^{16}$ The other cell-cell signaling system in $P$. aeruginosa functions through the AHQ signal-based system. ${ }^{14}$ The major AHQ signals include 2-heptyl-3hydroxy-4-quinolone, the Pseudomonas quinolone signal (PQS) and 2-heptyl-4-quinolone. ${ }^{14}$ These systems control the production of numerous virulence factors. ${ }^{18,19}$ On top of the QS systems, there is a complex regulatory network that controls the QS systems both at a transcriptional level and at a post-transcriptional level. ${ }^{16}$ Recently, a study demonstrated that QS system regulation was mediated by

\footnotetext{
${ }^{1}$ Key Laboratory of Resources Biology and Biotechnology in Western China, Ministry of Education, Faculty of Life Sciences, Northwest University, Xi'an, China and ${ }^{2}$ Department of Chemistry, The University of Chicago, Chicago, IL, USA

Correspondence: Dr H Liang, The University of Chicago, E 337 57th street, Chicago, IL, USA.

E-mail: hhliang@uchicago.edu
}

Received 14 June 2011; revised 24 September 2011; accepted 20 October 2011; published online 7 December 2011 
sub-inhibitory concentrations (SICs) of antibiotics and was a potential target for anti-infective therapy. ${ }^{20}$

Previously, we observed that SICs of antibiotics, including tetracycline (TET), activated phenazine production in P. aeruginosa, and we identified 32 transposon mutants that influenced expression of the phenazine synthesis gene (phzA1). Among them was the PA0011 mutant (LH02), in which phzA1 expression was increased about eightfold by TET compared with that in the wild-type PAO1. ${ }^{21}$ Here, we report that the PA0011 mutant is not only susceptible to an array of antibiotics but also involved in the regulation of the QS system.

\section{MATERIALS AND METHODS}

\section{Bacterial strains and growth conditions}

The bacterial strains and plasmids used in this study are listed in Table 1. P. aeruginosa $\mathrm{PAO} 1$ and derivatives were routinely grown at $37^{\circ} \mathrm{C}$ on $\mathrm{LB}$ (LuriaBertani) agar plates or in LB broth with shaking at 200 r.p.m. For E. coli, gentamicin $(\mathrm{Gm})$ was used at $15 \mu \mathrm{g} \mathrm{ml}^{-1}$, TET at $10 \mu \mathrm{g} \mathrm{ml}^{-1}$, kanamycin at $50 \mu \mathrm{g} \mathrm{ml}^{-1}$ and ampicillin at $100 \mu \mathrm{g} \mathrm{ml}^{-1}$. For P. aeruginosa, Gm was used at $50 \mu \mathrm{g} \mathrm{ml}^{-1}$ in LB and $150 \mu \mathrm{g} \mathrm{ml}^{-1}$ in Pseudomonas isolation agar, TET at $75 \mu \mathrm{g} \mathrm{ml}^{-1}$ in LB and $200 \mu \mathrm{g} \mathrm{ml}^{-1}$ in Pseudomonas isolation agar, carbenicillin was used at $500 \mu \mathrm{g} \mathrm{ml}^{-1}$ in Pseudomonas isolation agar and trimethoprim at $300 \mu \mathrm{g} \mathrm{ml}^{-1}$.

\section{DNA analysis and manipulation}

Restriction enzymes and DNA polymerase or T4 ligase were purchased from Fermentas or Takara and were used under the conditions recommended by the manufacturers. Plasmid DNA was isolated using a plasmid kit (Biotek, Beijing, China). Treatment of DNA with enzymes, subcloning of DNA and transformation of plasmids into E. coli and P. aeruginosa were carried out using standard methods. Where required, DNA fragments were isolated from agarose gels using a Fermentas gel extraction kit. Standard methods were used for the preparation of electro-competent cells and for plasmid electroporation into $E$. coli and P. aeruginosa. ${ }^{22}$ Nucleotide sequences were determined using a 377A DNA sequencer (Sangon, Shanghai). DNA sequences were compared with the BLASTN program using information from a Pseudomonas database (http://www.pseudomonas.com).

\section{Gene-replacement mutagenesis}

For gene replacement, the previously described sacB-based strategy was employed. ${ }^{23}$ The suicide plasmid, $\mathrm{pKD} 0011 \mathrm{~K}$, for gene replacement was constructed by inserting the BamHI-digested $\mathrm{Gm}^{\mathrm{r}}$-lacZ cassette derived from pZ1918-lacZ ${ }^{24}$ between two PCR fragments of PA0011 cloned into pEX18Ap. The $533 \mathrm{bp}$ upstream fragment of PA0011 was amplified using the primers, NH1 (5'-CGGCTGCAGTGCGTTCGGATGTTCCAC-3') containing a PstI site (underlined), and $\mathrm{NH} 2$ (5'-GCCGGATCCGCTTGGCGTAGGAGCAAT- $3^{\prime}$ ) containing a BamHI site (underlined). The $537 \mathrm{bp}$ downstream region of PA0011 was generated using primers, NH3 (5'-CGCGGATCCGCCCAAGCT

Table 1 Strains and plasmids used and constructed in this study

\begin{tabular}{|c|c|c|}
\hline Strain or plasmid & Relevant feature & Source or reference \\
\hline \multicolumn{3}{|l|}{ Strains } \\
\hline \multicolumn{3}{|l|}{$P$. aeruginosa } \\
\hline PA01 & Pseudomonas aeruginosa wild type & This lab \\
\hline LHO2 & PA0011 transposon mutant of PA01, $\mathrm{Gm}^{\mathrm{r}}$ & This study \\
\hline $\mathrm{PAO}(\Delta 0011)$ & PA0011 replacement mutant of PA01, PA0011:: $\mathrm{Gm}^{r}$ & This study \\
\hline $\mathrm{PAO}(\triangle 0011) \mathrm{C}$ & $\mathrm{PAO}(\Delta 0011)$ complemented strain & This study \\
\hline$\Delta p q s R$ & $p q s R$ replacement mutant of $\mathrm{PAO} 1, \mathrm{Gm}^{r}$ & This study \\
\hline$\Delta p q s H$ & pqsH replacement mutant of $\mathrm{PAO} 1, \mathrm{Gm}^{r}$ & This study \\
\hline \multicolumn{3}{|l|}{ E. coli } \\
\hline & F- mcrA $\Delta$ (mrr-hsdRMS-mcrBC)80dlacZ $\Delta$ M15 $\Delta$ lacX74 deoR recA1 & \\
\hline $\mathrm{DH} 5 \alpha$ & 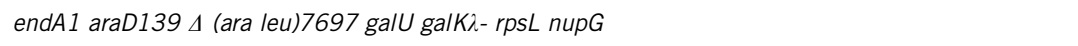 & Invitrogen \\
\hline Sm10 & Mobilizing strain, RP4 integrated in the chromosome; $\mathrm{Kn}^{\mathrm{r}}$ & 47 \\
\hline \multicolumn{3}{|l|}{ Plasmid } \\
\hline pEX18Ap & $\mathrm{oriT}^{+} \mathrm{sacB}^{+}$gene replacement vector with multiple-cloning site from $\mathrm{pUC18}, \mathrm{Ap}^{r}$ & 23 \\
\hline pZ1918lacZGm & Source plasmid of $\mathrm{Gm}^{\mathrm{r}}$ cassette & 24 \\
\hline pUCP28T & Broad-host-range vector & 48 \\
\hline pMS402 & Expression reporter plasmid carrying the promoterless IuxCDABE; $\mathrm{Kn}^{r} \mathrm{Tmp}^{r}$ & 26 \\
\hline \multirow[t]{2}{*}{ pKD-PA0011 } & pMS402 containing PA0011 promoter region & This study \\
\hline & PA0011 knock out plasmid pEX18Ap with 533 bp upstream region, Gm-lacZ fragment from & \\
\hline \multirow[t]{2}{*}{ pKD0011K } & pZ1918Gm-lacZ and 537 bp downstream of PA0011 cloned between Pstl and EcoRI; $\mathrm{Ap}^{r}\left(\mathrm{Cb}^{r}\right), \mathrm{Gm}^{r}$ & This study \\
\hline & Complementation plasmid, pUCP28T with a 1161 PCR fragment covering the entire & \\
\hline pKD0011C & PA0011 gene and 125 bp upstream and 65 bp downstream regions between HindIII and Pstl; Tmp ${ }^{r}$ & This study \\
\hline pKD-lasl & PMS402 containing las/ promoter region & 49 \\
\hline pKD-rhll & pMS402 containing $r$ hll promoter region & 49 \\
\hline pKD-lasR & pMS402 containing las $R$ promoter region & 49 \\
\hline pKD-rhIR & pMS402 containing $r h / R$ promoter region & 49 \\
\hline
\end{tabular}


GAAGGCAG-3') containing a BamHI site and NH4 (5'-GCGGAATTCGG GACCTGCGGAGGAG-3') containing an EcoRI site.

The plasmid, pKD0011K, was transferred to PAO1 using a tri-parental mating procedure employing the helper vector pRK2013. ${ }^{25}$ Briefly, overnight cultures $(25 \mathrm{ml}$ of each) of E. coli strains containing plasmids pKD0011K and pRK2013, respectively, and P. aeruginosa PAO1 were centrifuged and the pellets resuspended in phosphate-buffered saline, then mixed with equal portions and spotted onto an LB agar plate. Following incubation overnight at $37^{\circ} \mathrm{C}$, bacteria were removed from the agar surface and resuspended in $1 \mathrm{ml}$ of phosphate-buffered saline. Appropriate dilutions were plated onto Pseudomonas isolation agar containing $\mathrm{Gm}$ at $150 \mu \mathrm{g} \mathrm{ml}^{-1}$. Subsequently, strains that had undergone a second crossover event were selected for by plating on LB supplemented with $5 \%$ sucrose supplemented with $\mathrm{Gm}$. The PA0011 gene replacement mutant, designated $\mathrm{PAO}(\Delta 0011)$, was verified by assessing $\mathrm{Gm}$ resistance, carbenicillin sensitivity and PCR analysis.

\section{Complementation of the PA0011 mutant}

For the complementation experiments, PCR amplification was used to obtain a 1161-bp fragment of PA0011 containing its own promoter using the primers, 5'-GCG AAGCTTCATCTGCTACGCCTTCAT-3' and 5'-GCGCTGCAGCATAG TACCCGGATATG $-3^{\prime}$. Restrictions sites are shown as underlined, and were incorporated into the PCR primers. The fragment was digested with HindIII and PstI, and the generated fragment was subsequently ligated into a HindIIIPstI-digested broad-host-range vector, pUCP28T, to yield pKD0011C. The resultant pKD0011C was transformed into P. aeruginosa as described previously. ${ }^{22}$

\section{Construction of PA0011-luxCDABE}

The plasmid pMS402 carrying a promoterless luxCDABE reporter gene cluster was used to construct promoter-luxCDABE reporter fusions of the PA0011 operon as reported previously. ${ }^{21,26}$ The promoter regions of PA0011 were PCR amplified using high-fidelity $P f x$ DNA polymerase and primers synthesized according to the PAOl genome data. ${ }^{27}$ The primer pairs used were: $5^{\prime}$ CGCCTCGAGACCATCTGCTACGCCTT- ${ }^{\prime}$ and $5^{\prime}$-CGCGGATCCAGGTTG ATCCGCACTAC- $3^{\prime}$. The restriction sites, shown as underlined, were incorporated into the PCR primers. The promoter regions were cloned into the XhoIBamHI site upstream of the lux genes in pMS402. The constructs were transformed into PAO1 by electroporation. DNA manipulation, PCR and transformation were performed following general procedures. Cloned promoter sequences were confirmed by DNA sequencing.

\section{MIC determination}

To determine the MICs of antibiotics, overnight cultures were diluted such that the OD at $600 \mathrm{~nm}\left(A_{600}\right)$ was 0.2 , and cultivated for an additional $2 \mathrm{~h}$ before use as an inoculant. The cultures were inoculated into parallel wells of a 96-well plate with a transparent bottom. A $5-\mu l$ volume of the fresh cultures was seeded into the wells containing $195 \mu \mathrm{l}$ medium plus antibiotics in the first well, and the OD of the cultures adjusted to 0.07 by performing 1:1 serial dilutions in a 96-well microtiter plate. The OD of culture at $595 \mathrm{~nm}$ was measured every $30 \mathrm{~min}$ for $24 \mathrm{~h}$ in the Victor ${ }^{3}$ Multilabel Plate Reader (Perkin-Elmer, Waltham, MA, USA).

\section{Stress assays}

The susceptibilities of wild-type PAO1 and the PA0011 mutant strain to osmotic stress and heat shock were determined as previously described. ${ }^{1,28}$ In the heat shock experiments, cells were grown overnight in $\mathrm{LB}$ at $37^{\circ} \mathrm{C}$ and with aeration, then washed twice and diluted in $\mathrm{M} 9$ minimal medium to a density of $\sim 7000$ colony-forming units per $\mathrm{ml}\left(\mathrm{CFU} \mathrm{ml}^{-1}\right)$. One milliliter of the diluted culture was placed in a pre-warmed tube at $50{ }^{\circ} \mathrm{C}$, and the viability was determined by taking periodic aliquots and plating them directly onto LB agar plates. In order to measure sensitivity to osmotic stress, the strains were grown in $35 \mathrm{ml}$ of LB medium in $250 \mathrm{ml}$ flasks to an $A_{600}$ of 0.3 . At this point, the cultures were divided into two parts: one part was incubated with $\mathrm{NaCl}$ at a final concentration of $1.5 \mathrm{M}$; and the other was incubated without $\mathrm{NaCl}$.

\section{Luminescence screening assays}

Using these lux-based reporters, gene expression in liquid cultures was measured as counts per second (c.p.s.) of light production in a Victor ${ }^{3}$ Multilabel Plate Reader (Perkin-Elmer) or Synergy 2 (Biotek, Winooski, VT, USA), as previously described by our group. Overnight cultures of the reporter strains were diluted to an $A_{600}$ of 0.2 and cultivated for an additional $2 \mathrm{~h}$ before use. The cultures were inoculated into parallel wells of a black 96-well plate with a transparent bottom. A $5 \mu$ l volume of the fresh cultures was inoculated into the wells containing a total volume of $95 \mu \mathrm{l}$ medium plus other components, and the $A_{600}$ value in the wells was adjusted to around 0.07. A $60 \mu \mathrm{l}$ volume of filter-sterilized mineral oil was added to prevent evaporation during the assay. Promoter activities were measured every $30 \mathrm{~min}$ for $24 \mathrm{~h}$. Bacterial growth was monitored at the same time by measuring the OD at $595 \mathrm{~nm}$ in a Victor $^{3}$ Multilabel Plate Reader. Expression on solid medium was carried out by plating the reporter strains onto soft top LB agar and imaging in a LAS300 Imaging System (Fuji Corp., Tokyo, Japan).

\section{RESULTS}

\section{Antimicrobial susceptibility testing}

Our previous work has reported that expression of the phzAl operon in the LH02 strain is increased about eightfold compared with that in the wild-type PAO1 at $1 / 16 \mathrm{MIC}$ of TET. ${ }^{21}$ However, the growth rate of LH02 was inhibited when cultured in LB medium with 1/4 MIC of TET (Figure 1a). To address whether PA0011 serves as a possible target for antimicrobial agents, insertional inactivation of the PA0011 gene was performed in P. aeruginosa PAO1. The MICs of the PA0011 mutant and wild-type PAO1 are summarized in Table 2. The data showed that carbenicillin, TET, ciprofloxacin, chloramphenicol, polymixin, rifampin and erythromycin MICs for the PA0011 mutant were

a

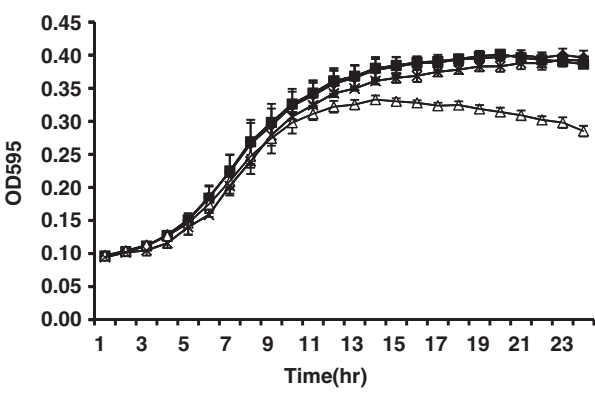

b PAO1
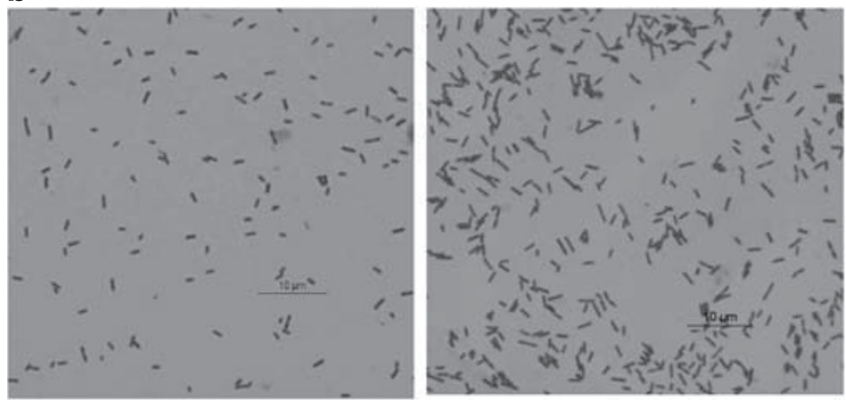

Figure 1 Growth profile and phenotype of wild-type PAO1 and the PA0011 mutant. (a) The growth of wild-type PAO1 and PAO1 $(\Delta 0011)$ in LB medium with or without $1.25 \mathrm{~g} \mathrm{ml}^{-1}$ TET. Wild-type PAO1 $(O)$ and PAO1 $(\Delta 0011)$ ( $\mathbf{a})$ cultured in LB. Wild-type PAO1 $(\triangle)$ and PAO1 $(\Delta 0011)(\boldsymbol{\nabla})$ in LB with $1.25 \mathrm{\mu g} \mathrm{ml}^{-1}$ TET. Values are means \pm standard deviations of triplicate experiments. The growth rate of mutant was considered to be statistically significant (Student's $t$-test, $P<0.005)$ compared with the wild-type PAO1. (b) Light microscopic images of crystal violet-stained cells at a magnification $\times 100$ after $16 \mathrm{~h}$ culture on LB agar plate. The average diameter of PAO $(1.65 \pm 0.43 \mu \mathrm{m})$ and $\mathrm{PAO} 1(\Delta 0011)(1.89 \pm 0.57 \mu \mathrm{m})$. 
at least twofold decreased from that for wild-type PAO1. As expected, the MICs of the complement PA0011 mutant was restored to wildtype PAO1 levels. Indeed, microscopic examination of cell size after staining with crystal violet revealed the diameter of the PA0011 mutant $(1.89 \pm 0.57 \mu \mathrm{m})$ was larger compared with wild-type PAO1 $(1.65 \pm 0.43 \mu \mathrm{m})$ (Figure $1 \mathrm{~b}$ ). These morphological patterns were similar to those observed when $P$. aeruginosa are exposed to SICs of cell wall-active antibiotics, such as $\beta$-lactams. ${ }^{29}$

\section{Response of PA0011 to environmental stress}

Our data showed that the PA0011 mutant was susceptible to numerous antibiotics. This made us wonder whether the PA0011 mutant was subject to other environmental stresses. The abilities of the PA0011 mutant and the wild-type PAO1 to resist drastic changes in temperature and osmolarity were investigated. Stationary-phase cultures of both strains were exposed to a sudden shift in temperature, $37-50{ }^{\circ} \mathrm{C}$. After $2-20 \mathrm{~min}$ at $50^{\circ} \mathrm{C}$, lethality in the PA0011 mutant cultures was higher than that in the parent cultures (Figure 2a). After $20 \mathrm{~min}$, the lethality of the mutant strain was about 20 times higher than the wild type. Exposure of the PA0011 gene rendered the cells more sensitive to both temperature and osmotic variations than its wild-type counterpart (Figure 2b). When the cells were subjected to an increase in osmotic pressure caused by the addition of a high concentration of salt, the PA0011 mutant was more sensitive (data not shown). These results indicate that the PA0011 gene responds to environmental stresses, such as temperature and osmotic variations.

Table 2 Antibiotic susceptibilities for the parent (PAO1), mutant and complement strains

\begin{tabular}{llcccccc}
\hline & \multicolumn{7}{c}{$\left.M I C(\mu g)^{-1}\right)$} \\
\cline { 2 - 8 } Strain & CIP & Tet & $C m$ & Poly & Rif & $C b$ & Em \\
\hline P. aeruginosa & $0.4 \pm 0.1$ & $10 \pm 2$ & $100 \pm 10$ & $3 \pm 1$ & $25 \pm 5$ & $125 \pm 15$ & $200 \pm 20$ \\
LHO2 & $0.1 \pm 0$ & $4 \pm 1$ & $30 \pm 5$ & $1.5 \pm 0.5$ & $10 \pm 2$ & $40 \pm 5$ & $90 \pm 10$ \\
PAO $(\Delta 0011)$ & $0.1 \pm 0$ & $3 \pm 1$ & $25 \pm 5$ & $1.5 \pm 0.5$ & $10 \pm 2$ & $40 \pm 5$ & $90 \pm 10$ \\
PAO $(\Delta 0011) C$ & $0.3 \pm 0.1$ & $8 \pm 1$ & $100 \pm 10$ & $3 \pm 1$ & $25 \pm 5$ & $125 \pm 10$ & $180 \pm 20$ \\
\hline
\end{tabular}

aAbbreviations: $\mathrm{Cb}$, carbenicillin; CIP, ciprofloxacin; $\mathrm{Cm}$, chloramphenicol; Em, erythromycin; Poly, polymixin; Rif, rifampin; Tet, tetracycline.
Expression of the PA0011::luxCDABE was activated by SICs of antibiotics

We have shown that the PA0011 gene was related to antibiotics and environmental stresses. On the basis of these observations, we speculated that the activation of the PA0011 gene may be regulated by antibiotics. To test this hypothesis, the promoter-reporter fusion plasmid of PA0011::luxCDABE was constructed and its activation investigated in the presence of antibiotics.

A disk-fusion method was initially used to visualize the response to antibiotics. The reporter strain carrying the PA0011 promoter was spread onto agar in a petri dish. The tested antibiotics were spotted onto filter discs laid on top of the agar. A positive response was observed as a change in light emission from the reporter strain beyond the zone of inhibition. The white light intensity reflected the promoter activity activated by these antibiotics, with the edge of the zone demonstrating inhibition ability. As shown in Figure 3a, the PA0011 promoter is activated by TET, streptomycin, azitromycin, erythromycin and ciprofloxacin. It is slightly activated by $\mathrm{Gm}$, chloramphenicol, spectinomycin but no activation was observed with polymyxin and rifampicin.

Further analysis of promoter regulation using SICs of antibiotics was carried out in multiwell plates and expression was measured as light production (c.p.s.) on a Multilabel Plate Reader. We used TET and erythromycin as the example to illustrate the effect at SICs of antibiotics on the activation of PA0011 in LB broth. The data showed that the activation of PA0011 was upregulated by about twofold at SICs of TET and erythromycin (Figure 3b). The activities observed were similar to that on the LB agar plate assay.

\section{Interaction between PA0011 and PQS}

Recent research has demonstrated that PQS does not follow the classical QS signal tenet, but is instead trafficked within the P. aeruginosa population via membrane vesicles. ${ }^{30}$ membrane vesicles are a bilayer with an outer leaflet of LPS $^{31}$ and an inner leaflet of phospholipids. LPS is composed of lipid A, core oligosaccharides and the polysaccharide O-antigen. ${ }^{32}$ Mazumder and colleagues ${ }^{30}$ have shown that PQS interacts with the LPS component of the outer membrane to initiate membrane vesicle formation in P. aeruginosa. On the basis of these observations, we hypothesized that PQS may affect the expression of the PA0011 gene. To determine the relationship between PA0011 and the PQS system, the activation of the promoterreporter fusion $\mathrm{pKD}-\mathrm{PA0011}$ was tested in wild-type $\mathrm{PAO} 1$ and $p q s R$,
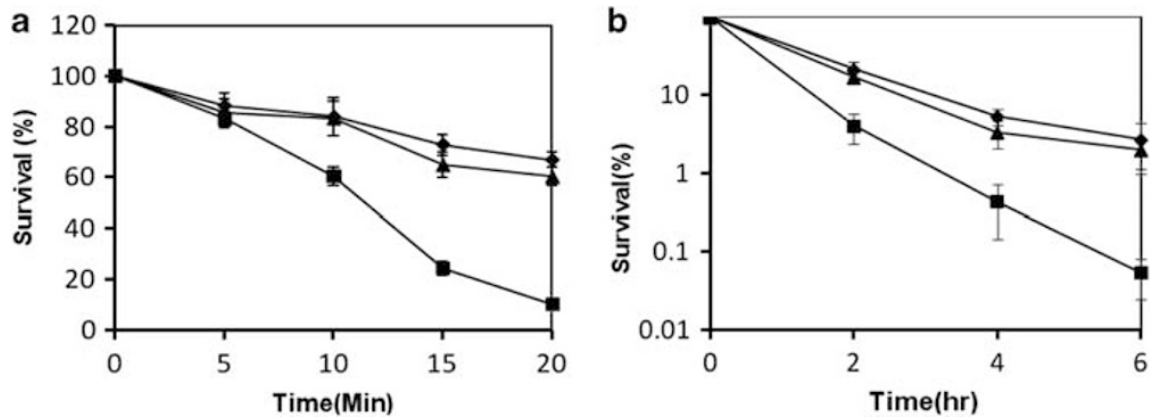

Figure 2 Susceptibility to temperature or osmotic stresses for the wild-type PAO1 and PA0011 mutant. (a) To assay cell survival of PAO1 ( $)$ ), PAO1 $(\triangle 0011) \mathrm{C}(\mathbf{\Lambda})$ and PAO1 $(\Delta 0011)$ ( $)$ strains after exposure to heat shock at $50^{\circ} \mathrm{C}$. Viability was expressed as a percentage of the number of $\mathrm{CFU}$ at time zero. (b) To assay cell survival of PAO1 $(\bullet)$, PAO1 $(\Delta 0011) \mathrm{C}(\mathbf{\Delta})$ and $\mathrm{PAO}(\Delta 0011)(\mathbf{\square})$ strains after exposure to $1.5 \mathrm{M} \mathrm{NaCl}$. Cell viability was determined after 2, 4 and $6 \mathrm{~h}$. One hundred percent viability corresponded to the number of CFU present immediately after resuspension of the cells in $1.5 \mathrm{~m} \mathrm{NaCl}$. Values are means \pm standard deviations of triplicate heat and osmotic shock experiments. 
a
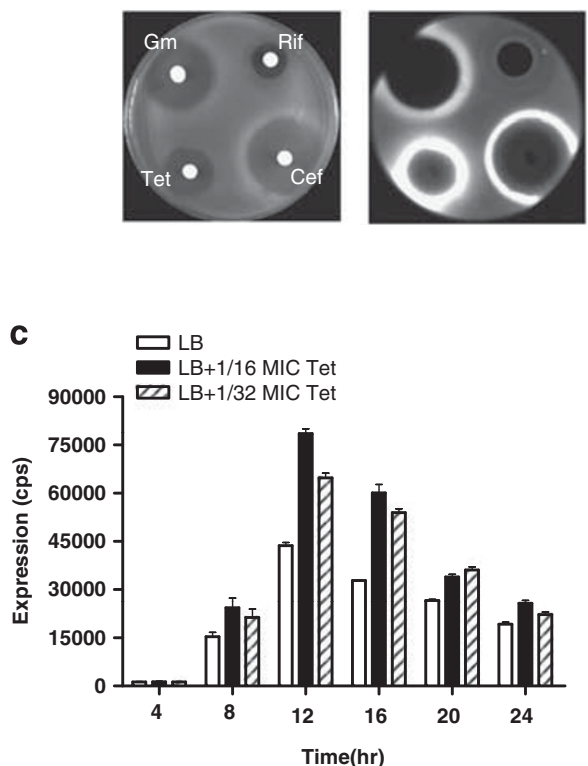

b
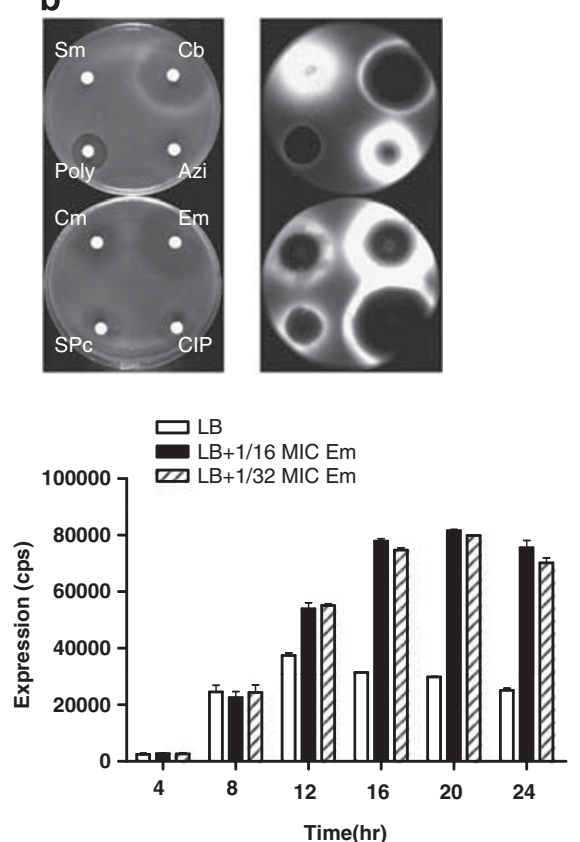

Figure 3 Activation of PA0011 in LB conditions with different antibiotics. (a, b) The PA0011 promoter was activated by antibiotics on solid plates. The left panel is a standard photograph of the plate showing the zones of inhibition. On the right is an image taken in the dark showing the specific activation of the PA0011 promoter by antibiotics. The white light intensity reflects the promoter activity, with the edge of the zone reflecting inhibition ability. (c) Expression profiles of the PA0011-/uxCDABE transcriptional fusion reporter in a 96-well plate assay. The plasmid, pKD-PA0011, was transformed into wild-type PAO1 and the strain cultured in LB (white bars) or LB with 1/16 (black bars) and 1/32 MIC (slash bars) TET and Em. Values are means \pm standard deviations of triplicate experiments. Azi, azitromycin; Cb, carbenicillin; CIP, ciprofloxacin; Cm, chloramphenicol; c.p.s., counts per second; Em, erythromycin; Poly, polymyxin; Sm, streptomycin; SPc, spectinomycin.
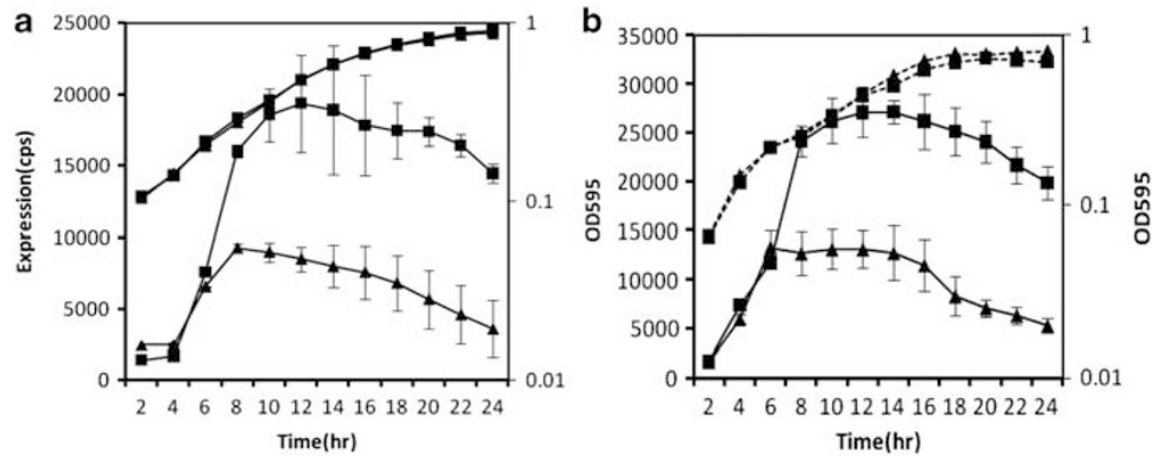

Figure 4 Expression profiles of PAOO11 in pqsR (a) and $p q s H(\mathbf{b})$ mutant strains. $P$. aeruginosa wild type ( $)$ and mutant strains ( $\mathbf{\square})$ containing the promoter-reporter fusion plasmid pKD-PA0011 cultured in LB medium. The corresponding growth curves are shown by dashed lines. The expression of pKD-PA0011 in mutants was considered to be statistically significant (Student's $t$-test, $P<0.005$ ) compared with the wild-type PAO1.

pqsH mutants, which are not capable of PQS production. The data showed the expression of PA0011 increased about twofold in $p q s R$ and pqsH mutants compared with the wild-type PAO1 (Figure 4).

Transcriptional expression of the QS genes in the PA0011 mutant Previous work has shown that the QS system is associated with multidrug resistance and biofilm formation in bacteria. ${ }^{33}$ Murakami et al. ${ }^{34}$ has also reported that the alternative sigma factor, RpoS, has a role in antibiotic tolerance in $P$. aeruginosa. Our data showed that the PA0011 gene was negatively regulated by the PQS system. Indeed, the PQS system is correlated to the las and $r h l$ systems. ${ }^{35}$ Based on these observations, we analyzed the expression of QS-genes in PA0011 mutant. The promoter-reporter fusions $p K D$-lasI, $p K D$-lasR, $p K D$ -
rhlI and pKD-rhlR were transformed into the PA0011 mutant and their expression was screened with a Multilabel Plate Reader Victor ${ }^{3}$ 1420. The results are shown in Figure 5. The activation of lasI, lasR and rhlI increased at least twofold at stationary phase in PA0011 mutant. Indeed, the activation of the QS-promoter at SICs of TET in the PA0011 mutant compared with wild type, exhibited a notable increase in QS expression (Figure 5), indicating PA0011 gene negatively regulates QS system. However, no differences of $p q s R$ and $p q s H$ were observed between them (data not shown).

\section{DISCUSSION}

In this study, we reported the isolation and characterization of a transposon mutant from a $P$. aeruginosa mutagenesis library. ${ }^{21}$ 

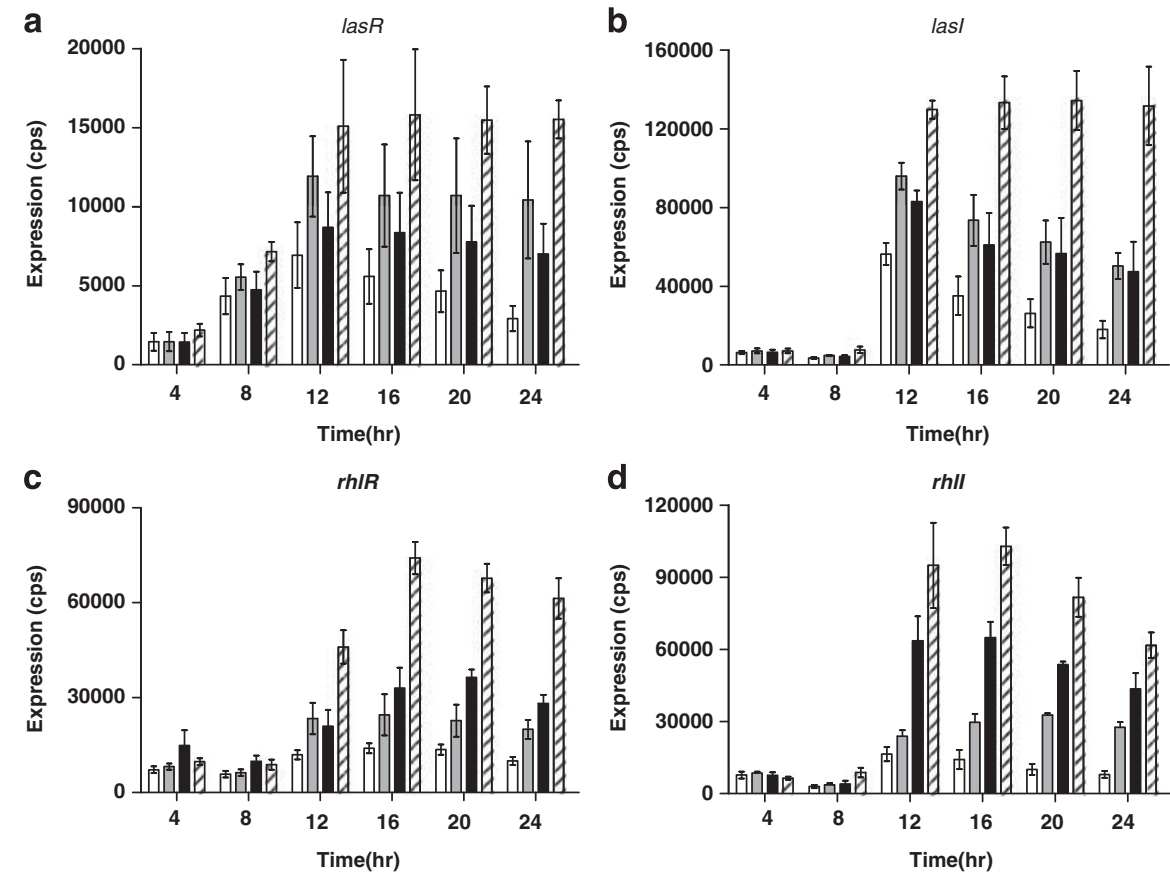

Figure 5 Expression profiles of the QS genes in wild-type PAO1 and the PA0011 mutant. The expression profiles of the promoter-reporter fusions of las $R$ (a), lasl (b), rhIR (c) and $r h l l$ (d) were measured every $30 \mathrm{~min}$. The cultures were placed in an aerobic jar and incubated at $37^{\circ} \mathrm{C}$. The gray and white bars

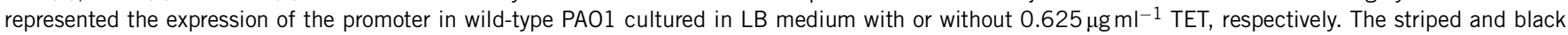
bars represent the expression of the promoter in PA0011 mutant cultured in LB medium with or without $0.625 \mu \mathrm{gml} \mathrm{m}^{-1} \mathrm{TET}$, respectively. Values are means \pm s.d. of triplicate experiments.

The PA0011 gene encodes 2-OH-lauroyltransferase, and we find the PA0011 mutant is more susceptible to a large variety of antibacterial agents than its parental strain PAO1 (Figure 1a, Table 1). The drug susceptibility of the $\operatorname{PAO}(\Delta 0011)$ strain was also accompanied by regulation of QS systems and reduced resistance to osmotic and heat shock stress. Indeed, the activation of PA0011 was induced by numerous antibiotics (Figure 3). These data presented suggest that the PA0011 mutant, depending on the growth phase and the nutritional balance in its environment, allows for different triggers of survival under stressful conditions, such as antibiotic addition.

Dotsch et al. ${ }^{36}$ has shown that 40 mutants were confirmed to be hyper-susceptible to several different antibiotics in $P$. aeruginosa. As we know, disrupting the mexA and mexB genes, which encode the major intrinsic multidrug MexAB-oprM efflux pump in $P$. aeruginosa, ${ }^{37}$ exhibited the most pronounced overall susceptibility. Insertions into the genes involved in cell envelope biogenesis resulted in hypersusceptibility, including $w z z$ gene, which is important for determining the length of the O-antigen side chain attached to LPS and influential in serum resistance. Indeed, genes encoding putative lipoprotein and outer membrane proteins, such as $\mathrm{OprF}, \mathrm{OmpH}$ and PA2800, were shown to be susceptible. In the present study, the PA0011 mutant was constructed and shown to be susceptible to a large variety of antibiotics compared with the wild-type PAO1 (Table 2). The PA0011 mutant strain was also sensitive to $1.5 \mathrm{M} \mathrm{NaCl}$ and heat shock (Figure 2). The PA0011 gene encodes 2-OH-lauroyltransferase that is required for 3-deoxy-D-manno-octulosonic acid (Kdo) addition in lipid A biosynthesis and can rapidly incorporate one laurate residue into (Kdo) ${ }_{2}$-lipid IVA. ${ }^{38}$ Moreover, outer membrane proteins are key molecules with regard to the interface between the cell and its environment, and these proteins have been suggested to influence the intrinsic resistance to antibiotics. Using a proteomic approach, a Vibrio vulnificus ompH mutant showed enhanced sensitivity to SDS and polymyxin B. ${ }^{39}$ Therefore, all the altered phenotypes in the PA0011 mutant may be due to deficiencies of lauroytransferase influence during synthesis of the cell membrane. The detailed mechanism needs to be investigated in future.

Recent antimicrobial research has focused on induction of the SOS response by antibiotics and the role that SOS response has in resistance development. ${ }^{40}$ In addition to the well-characterized role of inhibitory concentrations, SICs of antibiotics may also be critical in this context. In our previous work, a transposon mutagenesis library was used to examine the effects at SICs of TET on virulence gene expression in $P$. aeruginosa. ${ }^{21}$ Moreover, this was expended here with the addition of TET and concentrations of other antibiotics. Interestingly, the expression of QS-controlled genes, such as phzAl and rhlA (data not shown) and Rhl-QS genes in PA0011 mutant, were upregulated in condition with TET (Figure 5). Skindersoe et al. ${ }^{41}$ showed that TET had little effect on QS, but that low concentrations of azithromycin, ceftazidime and ciprofloxacin have QS inhibitory activity in P. aeruginosa. Indeed, previous studies have shown that SICs of ceftazidime, tobramycin and azithromycin reduce the QS signals in P. aeruginosa. ${ }^{42,43}$ In contrast, our results indicated that SICs of TET significantly activated the expression of QS-controlled genes in PA0011 mutant. Accordingly, we assumed that PA0011 may act as a receptor to influence the expression of virulence factors in the presence of SICs antibiotics.

QS in P. aeruginosa consists of a complex network and there are at least three QS systems; that is, las, $r h l$ and AHQ. ${ }^{16}$ A hierarchy within the $N$-acyl-HSL-based QS system is believed to exist in which the las system controls the rhl system. PQS, as one of the signals of AHQ, is intricately connected to the AHL systems. ${ }^{15}$ The $r h l$ and las systems, respectively, exert negative and positive regulation on PQS, whereas PQS positively influences the $r h l$ system. PqsR(MvfR) is the response regulator in the PQS system. ${ }^{44}$ The results have shown that the 
expression of las and rhl was affected in the PA0011 mutant (Figure 5). Indeed, the PA0011 promoter activity was increased in the $p q s R$ and $\mathrm{pqsH}$ mutant strains (Figure 4). Therefore, it is possible that the PQS system regulates the rhlllas systems via the PA0011 gene.

Increasingly, a global concern has emerged that infections controlled by interfering with QS systems can be effective, and QS has been used as a target when people discover new antibiotics. ${ }^{45,46}$ Effective means to attenuate or inhibit pathogenicity involve in controlling or disturbing expression of QS systems. ${ }^{20}$ Characteristics of the newly identified mutant, PA0011, show that it is sensitive to an array of antibiotics, and contributes to QS systems expression. Therefore, it may enable researchers to elucidate new antibiotic targets in P. aeruginosa.

\section{ACKNOWLEDGEMENTS}

We thank our lab members for critically reading the manuscript. This work was supported by Project supported by the National Science Foundation for Young Scientists of China (Grant No. 31000049), Supported by the Natural Science Basis Research Plan in Shaanxi Province of China (Grant No. 2010JQ3008), the Scientific Research Foundation of the Education Department of ShaanXi Province (Grant No. 2010JK851), the NWU Doctorate Dissertation of Excellence Funds (Grant No. 08YYB05) and the Natural Science Foundation of NWU (Grant No. 09NW17).

1 Ramos-Aires, J., Plesiat, P., Kocjancic-Curty, L. \& Kohler, T. Selection of an antibiotichypersusceptible mutant of Pseudomonas aeruginosa: identification of the GImR transcriptional regulator. Antimicrob Agents Chemother 48, 843-851 (2004).

2 Gomez, M. J. \& Neyfakh, A. A. Genes involved in intrinsic antibiotic resistance of Acinetobacter baylyi. Antimicrob Agents Chemother 50, 3562-3567 (2006).

$3 \mathrm{Ma}$, D., Cook, D. N., Hearst, J. E. \& Nikaido, H. Efflux pumps and drug resistance in Gram-negative bacteria. Trends Microbiol 2, 489-493 (1994).

4 Li, X. Z., Ma, D., Livermore, D. M. \& Nikaido, H. Role of efflux pump(s) in intrinsic resistance of Pseudomonas aeruginosa: active efflux as a contributing factor to betalactam resistance. Antimicrob Agents Chemother 38, 1742-1752 (1994).

5 Nikaido, H. Prevention of drug access to bacterial targets: permeability barriers and active efflux. Science 264, 382-388 (1994).

6 Angus, B. L., Carey, A. M., Caron, D. A., Kropinski, A. M. \& Hancock, R. E. Outer membrane permeability in Pseudomonas aeruginosa: comparison of a wild-type with an antibiotic-supersusceptible mutant. Antimicrob Agents Chemother 21, 299-309 (1982).

7 Benz, R. \& Hancock, R. E. Properties of the large ion-permeable pores formed from protein $\mathrm{F}$ of Pseudomonas aeruginosa in lipid bilayer membranes. Biochim Biophys Acta 646, 298-308 (1981).

8 Kadrmas, J. L., Brozek, K. A. \& Raetz, C. R. Lipopolysaccharide core glycosylation in Rhizobium leguminosarum. An unusual mannosyl transferase resembling the heptosyl transferase I of Escherichia coli. J Biol Chem 271, 32119-32125 (1996)

9 Reeves, P. R. et al. Bacterial polysaccharide synthesis and gene nomenclature. Trends Microbiol 4, 495-503 (1996).

10 Singh, S. P., Miller, S., Williams, Y. U., Rudd, K. E. \& Nikaido, H. Immunochemical structure of the OmpD porin from Salmonella typhimurium. Microbiology 142 (Part 11), 3201-3210 (1996).

11 Gibson, R. L., Burns, J. L. \& Ramsey, B. W. Pathophysiology and management of pulmonary infections in cystic fibrosis. Am J Respir Crit Care Med 168, 918-951 (2003).

12 Hooper, D. C. Mechanisms of fluoroquinolone resistance. Drug Resist Updat 2, 38-55 (1999).

13 Winzer, K. \& Williams, P. Quorum sensing and the regulation of virulence gene expression in pathogenic bacteria. Int J Med Microbiol 291, 131-143 (2001).

14 Diggle, S. P., Cornelis, P., Williams, P. \& Camara, M. 4-Quinolone signalling in Pseudomonas aeruginosa: old molecules, new perspectives. Int J Med Microbiol 296, 83-91 (2006).

15 Diggle, S. P., Crusz, S. A. \& Camara, M. Quorum sensing. Curr Biol 17, R907-R910 (2007).

16 Williams, P., Winzer, K., Chan, W. C. \& Camara, M. Look who's talking: communication and quorum sensing in the bacterial world. Philos Trans $R$ Soc Lond B Biol Sci $\mathbf{3 6 2}$, 1119-1134 (2007)

17 von Bodman, S. B., Willey, J. M. \& Diggle, S. P. Cell-cell communication in bacteria: united we stand. J Bacteriol 190, 4377-4391 (2008).

18 Pearson, J. P., Pesci, E. C. \& Iglewski, B. H. Roles of Pseudomonas aeruginosa las and $\mathrm{rhl}$ quorum-sensing systems in control of elastase and rhamnolipid biosynthesis genes. J Bacteriol 179, 5756-5767 (1997).
19 Pearson, J. P., Passador, L., Iglewski, B. H. \& Greenberg, E. P. A second N-acylhomoserine lactone signal produced by Pseudomonas aeruginosa. Proc Natl Acad Sci USA 92, 1490-1494 (1995).

20 Finch, R. G., Pritchard, D. I., Bycroft, B. W., Williams, P. \& Stewart, G. S. Quorum sensing: a novel target for anti-infective therapy. J Antimicrob Chemother 42, 569-571 (1998).

21 Liang, H., Li, L., Dong, Z., Surette, M. G. \& Duan, K. The YebC family protein PA0964 negatively regulates the Pseudomonas aeruginosa quinolone signal system and pyocyanin production. J Bacteriol 190, 6217-6227 (2008).

22 Smith, A. W. \& Iglewski, B. H. Transformation of Pseudomonas aeruginosa by electroporation. Nucleic Acids Res 17, 10509 (1989).

23 Hoang, T. T., Karkhoff-Schweizer, R. R., Kutchma, A. J. \& Schweizer, H. P. A broadhost-range FIp-FRT recombination system for site-specific excision of chromosomallylocated DNA sequences: application for isolation of unmarked Pseudomonas aeruginosa mutants. Gene 212, 77-86 (1998).

24 Schweizer, H. P. Two plasmids, X1918 and Z1918, for easy recovery of the xylE and lacZ reporter genes. Gene 134, 89-91 (1993).

25 Ditta, G., Stanfield, S., Corbin, D. \& Helinski, D. R. Broad host range DNA cloning system for Gram-negative bacteria: construction of a gene bank of Rhizobium meliloti. Proc Natl Acad Sci USA 77, 7347-7351 (1980).

26 Duan, K., Dammel, C., Stein, J., Rabin, H. \& Surette, M. G. Modulation of Pseudomonas aeruginosa gene expression by host microflora through interspecies communication. Mol Microbiol 50, 1477-1491 (2003).

27 Stover, C. K. et al. Complete genome sequence of Pseudomonas aeruginosa PA01, an opportunistic pathogen. Nature 406, 959-964 (2000).

28 Suh, S. J. et al. Effect of rpoS mutation on the stress response and expression of virulence factors in Pseudomonas aeruginosa. J Bacteriol 181, 3890-3897 (1999).

29 Waisbren, S. J., Hurley, D. J. \& Waisbren, B. A. Morphological expressions of antibiotic synergism against Pseudomonas aeruginosa as observed by scanning electron microscopy. Antimicrob Agents Chemother 18, 969-975 (1980).

30 Mashburn, L. M. \& Whiteley, M. Membrane vesicles traffic signals and facilitate group activities in a prokaryote. Nature $437,422-425$ (2005).

31 Mazumder, R., Phelps, T. J., Krieg, N. R. \& Benoit, R. E. Determining chemotactic responses by two subsurface microaerophiles using a simplified capillary assay method. J Microbiol Methods 37, 255-263 (1999).

32 Fiocca, R. et al. Release of Helicobacter pylori vacuolating cytotoxin by both a specific secretion pathway and budding of outer membrane vesicles. Uptake of released toxin and vesicles by gastric epithelium. J Pathol 188, 220-226 (1999).

33 Pumbwe, L., Skilbeck, C. A. \& Wexler, H. M. Presence of quorum-sensing systems associated with multidrug resistance and biofilm formation in Bacteroides fragilis. Microb Ecol 56, 412-419 (2008).

34 Murakami, K. et al. Role for rpoS gene of Pseudomonas aeruginosa in antibiotic tolerance. FEMS Microbiol Lett 242, 161-167 (2005)

35 Diggle, S. P. et al. The Pseudomonas aeruginosa quinolone signal molecule overcomes the cell density-dependency of the quorum sensing hierarchy, regulates $r \mathrm{hl}$-dependent genes at the onset of stationary phase and can be produced in the absence of LasR. Mol Microbiol 50, 29-43 (2003).

36 Dotsch, A. et al. Genomewide identification of genetic determinants of antimicrobial drug resistance in Pseudomonas aeruginosa. Antimicrob Agents Chemother 53, 2522-2531 (2009).

$37 \mathrm{Li}$, X. Z., Nikaido, H. \& Poole, K. Role of mexA-mexB-oprM in antibiotic efflux in Pseudomonas aeruginosa. Antimicrob Agents Chemother 39, 1948-1953 (1995).

38 Clementz, T., Bednarski, J. J. \& Raetz, C. R. Function of the htrB high temperature requirement gene of Escherchia coli in the acylation of lipid A: HtrB catalyzed incorporation of laurate. J Biol Chem 271, 12095-12102 (1996).

39 Alice, A. F., Naka, H. \& Crosa, J. H. Global gene expression as a function of the iron status of the bacterial cell: influence of differentially expressed genes in the virulence of the human pathogen Vibrio vulnificus. Infect Immun 76, 4019-4037 (2008).

40 Mesak, L. R., Miao, V. \& Davies, J. Effects of subinhibitory concentrations of antibiotics on SOS and DNA repair gene expression in Staphylococcus aureus. Antimicrob Agents Chemother 52, 3394-3397 (2008)

41 Skindersoe, M. E. et al. Effects of antibiotics on quorum sensing in Pseudomonas aeruginosa. Antimicrob Agents Chemother 52, 3648-3663 (2008).

42 Garske, L. A., Beatson, S. A., Leech, A. J., Walsh, S. L. \& Bell, S. C. Sub-inhibitory concentrations of ceftazidime and tobramycin reduce the quorum sensing signals of Pseudomonas aeruginosa. Pathology 36, 571-575 (2004).

43 Tateda, K., Standiford, T. J., Pechere, J. C. \& Yamaguchi, K. Regulatory effects of macrolides on bacterial virulence: potential role as quorum-sensing inhibitors. Curr Pharm Des 10, 3055-3065 (2004).

$44 \mathrm{Cao}, \mathrm{H}$. et al. A quorum sensing-associated virulence gene of Pseudomonas aeruginosa encodes a LysR-like transcription regulator with a unique self-regulatory mechanism. Proc Natl Acad Sci USA 98, 14613-14618 (2001).

45 Rasmussen, T. B. \& Givskov, M. Quorum-sensing inhibitors as anti-pathogenic drugs. Int J Med Microbiol 296, 149-161 (2006).

46 Hentzer, M. et al. Attenuation of Pseudomonas aeruginosa virulence by quorum sensing inhibitors. EMBO J 22, 3803-3815 (2003).

47 Simon, R., Priefer, U. \& Pühler, A. A broad host range mobilization system for in vivo genetic engineering: transposon mutagenesis in Gram negative bacteria. Biotechnology 1, 784-791 (1983).

48 Schweizer, H. P. Allelic exchange in Pseudomonas aeruginosa using novel ColE1-type vectors and a family of cassettes containing a portable oriT and the counter-selectable Bacillus subtilis sacB marker. Mol Microbiol 6, 1195-1204 (1992).

49 Duan, K. \& Surette, M. G. Environmental regulation of Pseudomonas aeruginosa PAO1 Las and Rhl quorum-sensing systems. J Bacteriol 189, 4827-4836 (2007). 